\title{
SMART ELECTRONIC STICK FOR BLIND PEOPLE: AN IOT APPLICATION
}

\author{
Ch Sudhakar ${ }^{1}$, N. Thirupathi Rao ${ }^{1}$ and Debnath Bhattacharyya ${ }^{1}$ \\ ${ }^{1}$ Department of Computer Science \& Engineering \\ Vignan's Institute of Information Technology (A) \\ Visakhapatnam, AP, India \\ nakkathiru@gmail.com,debnathb@gmail.com
}

\begin{abstract}
The vision of the people gives an opportunity to see the surrounding of a human being and the surrounded world around the human being. Till today, the people who lost their vision due to several reasons are struggling to get a useful device or an article such that to help them in their day to day life. If we get success in preparing such a device can help them a lot in their lives. In the current article, we developed a cheap, friendly and operation simple tool that can benefit both the users like visually challenged and the impaired people such that they can use in their life. This device helps such people to use this device by having a wearable device like the wearable head cap and hand stick such that the impaired people can walk on the roads without the help of any other persons or other persons support. The blind people can walk of their own, and the device will guide the person to maintain some distance from the objects that were present on the way of the blind people on which they are going. The principal part of this framework is the ultrasonic sensor which is utilized to examine a foreordained territory around daze by transmitting reflecting waves. The reflected signs got from the boundary objects are being used as contributions to Arduino Uno microcontroller. The microcontrollers carry out the issued commands and then convey the obstacle message to the user through headphones. So, by listening to the authorities by microcontroller, the blind can walk safely on roads.
\end{abstract}

\section{Keywords - Arduino Microcontroller, Ultrasonic sensor, IoT}

\section{INTRODUCTION}

Visual impairment is a condition with which the person cannot see anything with his /her eyes which occurs because of physiological or neurological elements. Envision strolling into a new place. One needs to request direction with a specific end goal to reach to the target. Individual needs to rely upon other individuals to achieve the goal [1]. By and large, we watch that white stick is the closest companion of the outwardly hindered individual. But in reality, numerous times this stick isn't helpful. In a new encompassing blocked individual may get confounded. So, this limits their portability. This makes them subject to others for help. Despite the instrument utilized, the factor that most decides a man's portability is the utilization of fundamental individual abilities. Total visual weakness is the complete absence of frame and visible light wisdom and is clinically recorded as NLP, a shortening as "no light acknowledgement". Visual inadequacy is as frequently as conceivable used to portray extraordinary visual shortcoming with excellent vision [2].

Various people encounter the problems of honest to goodness visual impedances shielding them from voyaging independently. They need to use a broad assortment of gadgets and strategies to help them in their adaptability [3]. One of these systems is presentation and adaptability ace, which helps the ostensibly weakened and stupor people and prepares them to continue ahead their own independently and safely depending upon

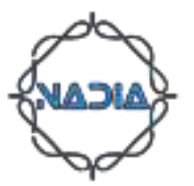


their other extraordinary resources. Another method is the guide mutts which are arranged remarkably to help the outwardly hindered people on their improvement by investigating around the hindrances to caution the person to change his/her way. In any case, this system has a couple of repressions, inconvenience to understand the brain-boggling course by these puppies, and they are sensible for around five years [4].

The cost of these prepared mutts is exceptionally costly likewise it is troublesome for a large number of visually impaired and outwardly impeded people to give the necessary care to another living being [5]. This gadget is light, convenient, yet extend restricted to its particular size, and it isn't usable for effective deterrents discovery neither than obstructions not situated on the floor. Starting late, various procedures have been made to update the convenience of outwardly disabled people that rely upon signal taking care of and sensor advancement. These called Electronic Travel Aid (ETA) devices assist the incognizant concerning move wholeheartedly in a space paying little regard to its dynamic changes.

According to the composition, these devices are generally portrayed into two vital perspectives: sonar input (laser hail, infrared signs, or ultrasonic signs) and camera input structures (includes primarily of a littler than anticipated CCD camera). The way these devices work mostly like the radar system that usages ultrasonic fascicle or laser to perceive stature, the bearing, and speed of settled and moving articles [6]. The partition between the individual and the blocks are assessed when of the wave travel. Regardless, every current structure instructs the outwardly disabled of the closeness of an inquiry at a specific detachment previously or near him. These points of interest allow the client to change his or her way. Data about the question qualities can make new learning to upgrade space appearance and memory of the visually impaired. To defeat the previously mentioned restrictions, this work offers a straightforward, productive, configurable virtual for the visually impaired [7].

\section{RELATED WORK}

The literature survey was led in two sections; in the first step the handheld and other for the non-hand, extensive research was done on the web hunting down licenses and sites for the different guides for the outwardly hindered. GPS helps and blind guides are additionally considered for the writing audit as takes after:

The handheld ultrasonic guides, generally are a significant decent guide for the outwardly impeded. They are sensibly cheap, simple to utilise and dependable. Notwithstanding, their one noteworthy ruin is that all together for remedy task of this guide, two hands are required. Be that as it may, they are incredibly costly. The least expensive is $\$ 980$, and they can be up to $\$ 2,000$. Unless governments can give some endowment, this is excessively costly for most visions debilitated clients. Remember $90 \%$ of them originate from under developed nations. The most broadly utilized and prescribed stick worked ultrasonic sensor is the Ultra cane. It is so delicate and the repair works are costly and some other problems identified, it has to be sent to the UK and the process is more time taking.

These are very vast, dark shades which have a sensor in the extension of the nose with vibrational yield are distinguished. Criticism from representatives of the RSB says this item is excessively apparent and sci-fi looking. Most outwardly weakened clients won't utilise this item accordingly [8]. GPS helps a generally new advancement in outwardly disabled items and are a better than average route for an outwardly weakened client to explore their way around vast zones around a city. Nonetheless, they are not as valuable for deciding impediments on a small scale level like a low entryway or a branch of a tree. Henceforth, this isn't such a significant asset for small-scale route and is better utilized for full-scale route. It has been found that this framework does not work so well inside or in swarmed urban communities in light of the GPS framework that it utilises (LA Pierre CM 
1998). It is clear then that this sort of innovation isn't exactly suited to this extent however it ought to be remembered so it can be investigated if the potential emerges later on.

In the blind guides, there is a guide called the C-5 laser stick [8,9]. This high sticks is an exceptionally costly guide and it isn't attractive because of the short battery life, which can be exceptionally severely arranged. The other guide is an electronic guide which utilises a 3D time of flight sensor; there is minimal referred to about this guide as it at present has not been created, at this phase there is just a patent on it. This, in principle, sounds like a decent alternative however until there is more data accessible, it is difficult to tell.

\section{PROPOSED SYSTEM}

To defeat the challenges in the current techniques available in the market and to give the practical and easy to use framework for daze route, the current plan is proposed. The underneath figure demonstrates that this undertaking and it comprises of five sections to be the specific Power supply, SD Card, Headphone, Microcontroller, Ultrasonic sensors.

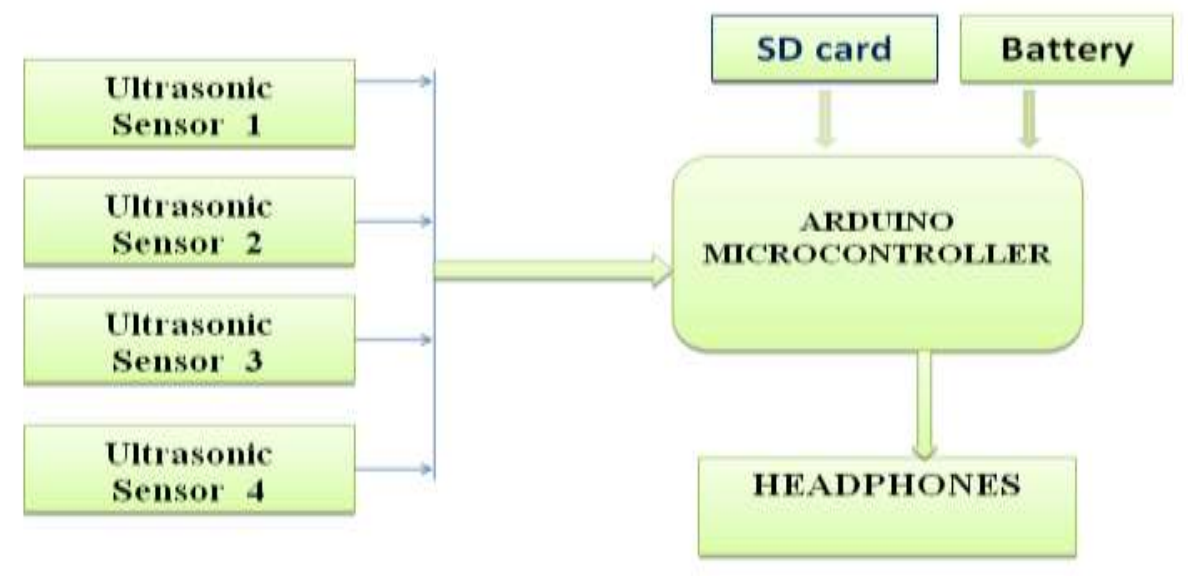

Fig. 1 Block Diagram of the smart electronic stick

Here Figure 1 shows there are four ultrasonic sensors. These sensors detect the objects up to 1.2 meters and send those signals to the Arduino microcontroller and Arduino microcontroller sends the obstacle message to the speakers or headphones.

\subsection{HARDWARE DESCRIPTION}

\section{ULTRASONIC SENSOR}

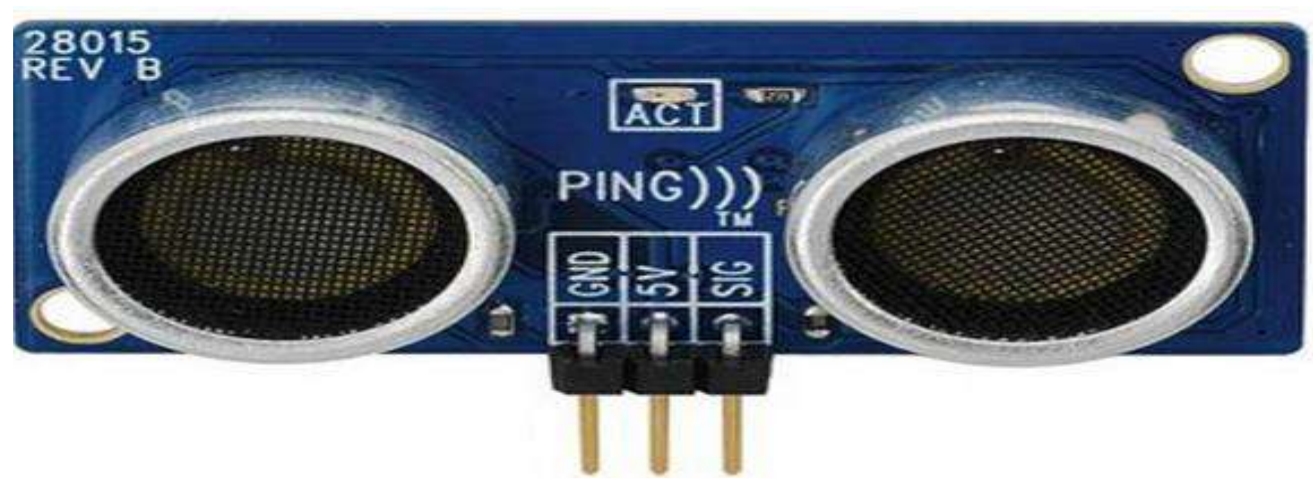

Fig. 2 Ultrasonic sensor 
The primary goal of using the ultrasonic transducer is to avoid the obstacles that were being present on the way of the blind people. This sensor unit can work for the transmission for the signals and for receiving the messages may work from the range of 2 centimetres to 120 centimetres that were the estimation of work. If it is extended further, it can work for another more 3 centimetres. This unit consists of the various transmitters with ultrasonic sensors connected, the control circuit for the entire group. This high unit uses a minimum input-output trigger for transmitting and working of the data, and the trigger takes place at every ten microseconds of time of various state signals. The sensor form the stick will send data continuously from the unit at every 10 microseconds of time. The sensor unit will transmit the data continuously from the range of $40 \mathrm{KHz}$, and motion back will take place.

The current sensor will produce the sound by giving the data to the obstacle, and the separation of the surface can be identified easily and the sound waves will be generated and also it will estimate the time will take for reaching the target object. The sensor unit can identify the object before to the distance from 3 centimetres to the range of 3 meters approximately. The primary sensor will consist of two parts in the unit such that it will work based on the functioning of these two units. They are a producer who can produce the signals of strength around $40 \mathrm{kHz}$ sound waves, and the other device unit was the finder, i.e., the object finder. To decide the separation to a protest, it is essential to execute a planning circle in your microcontroller code for the divorce to the object.

\section{ARDUINO MICROCONTROLLER}

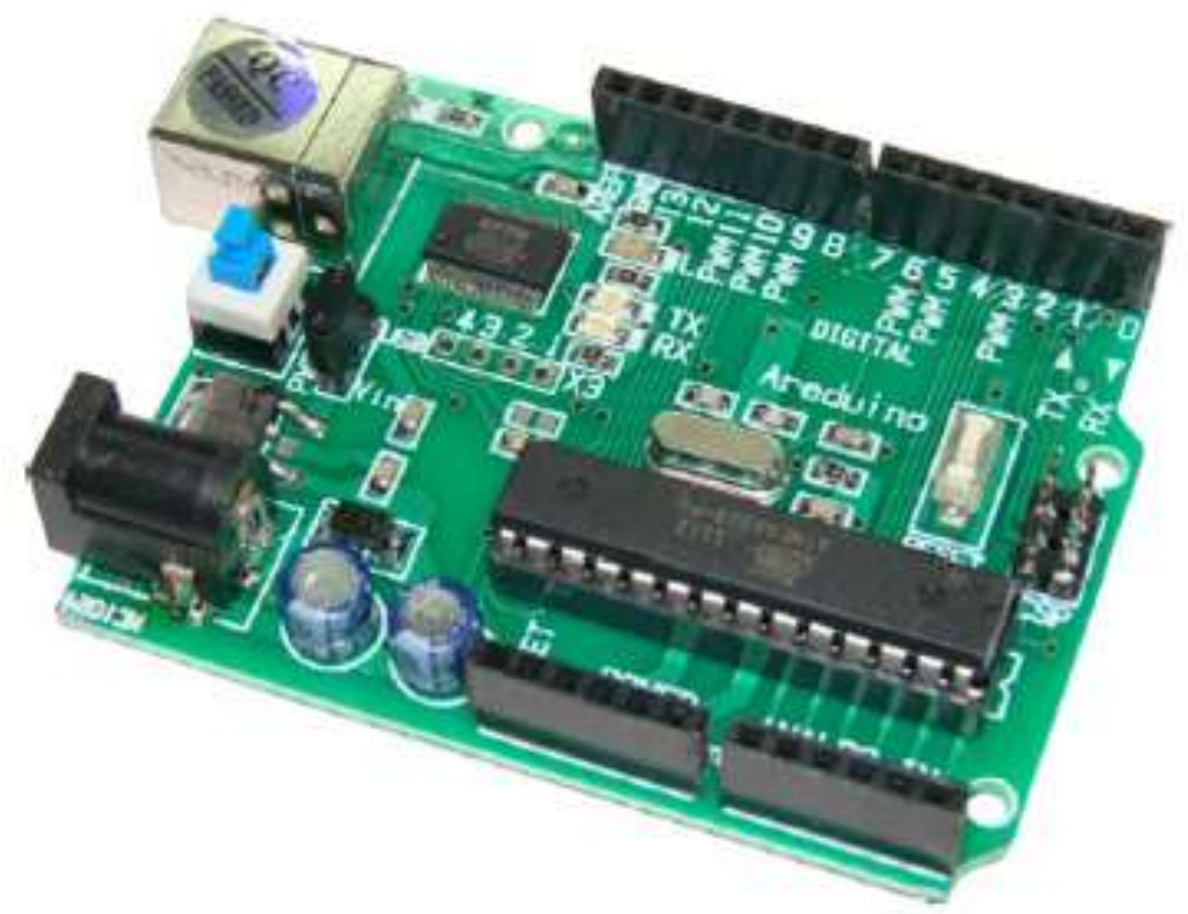

Fig. 3 Arduino Microcontroller

Arduino is a single board microcontroller board which was considered to utilise for the usage of designing and working with the automatic devices or the applets which can be needed to be controlled by the other units like the remote controls or with the mobile devices from various locations. The hardware unit for the Arduino microcontroller can be seen from the above image, and it will consist of an 8-bit Atmel microcontroller. In some cases, the users can use the Atmel ARM microcontroller which will work with a 32- bit 
help. The current day units of this Arduino may have the USB interface such that any device can be connected to this particular unit. It consists of around six pins in straight and also inputs output pins around 14. These pins will help the customer to connect the group with another mode of devices and can work with the current Arduino unit. This unit will consist of a diamond mode oscillator. This unit will have a USB jack unit and also the power jack such that to connect with other devices. It also will have a reset button such that to reset the group at any time to make it reboot.

\section{OBSTACLE DETECTION ALGORITHM}

STEP 1: Start

STEP 2: Declare ultrasonic sensors as rs, ls, fs, bs.

STEP 3: Initialize the values of rs, fs, bs, ls to 0.

STEP 4: If an any one of the sensor senses obstacle and its distance is less than 50 then go to next step.

STEP 5: If rs distance is less than 50 then the controller sends the message to headphones as "obstacle please turn left".

STEP 6: If bs distance is less than 50 then the controller sends the message to headphones as "obstacle please turn right".

STEP 7: If fs distance is less than 50 then the controller sends the message to headphones as "obstacle please come back".

STEP 8: If ls distance less than 50 then the controller sends the message to Headphones as "obstacle please goes forward".

STEP 9: If two or more sensors distance is less than 50 then the controller sends the message to headphones as "obstacle".

STEP10: Stop.

where rs- right sensor, ls- left sensor, fs- front sensor, bs- back sensor 


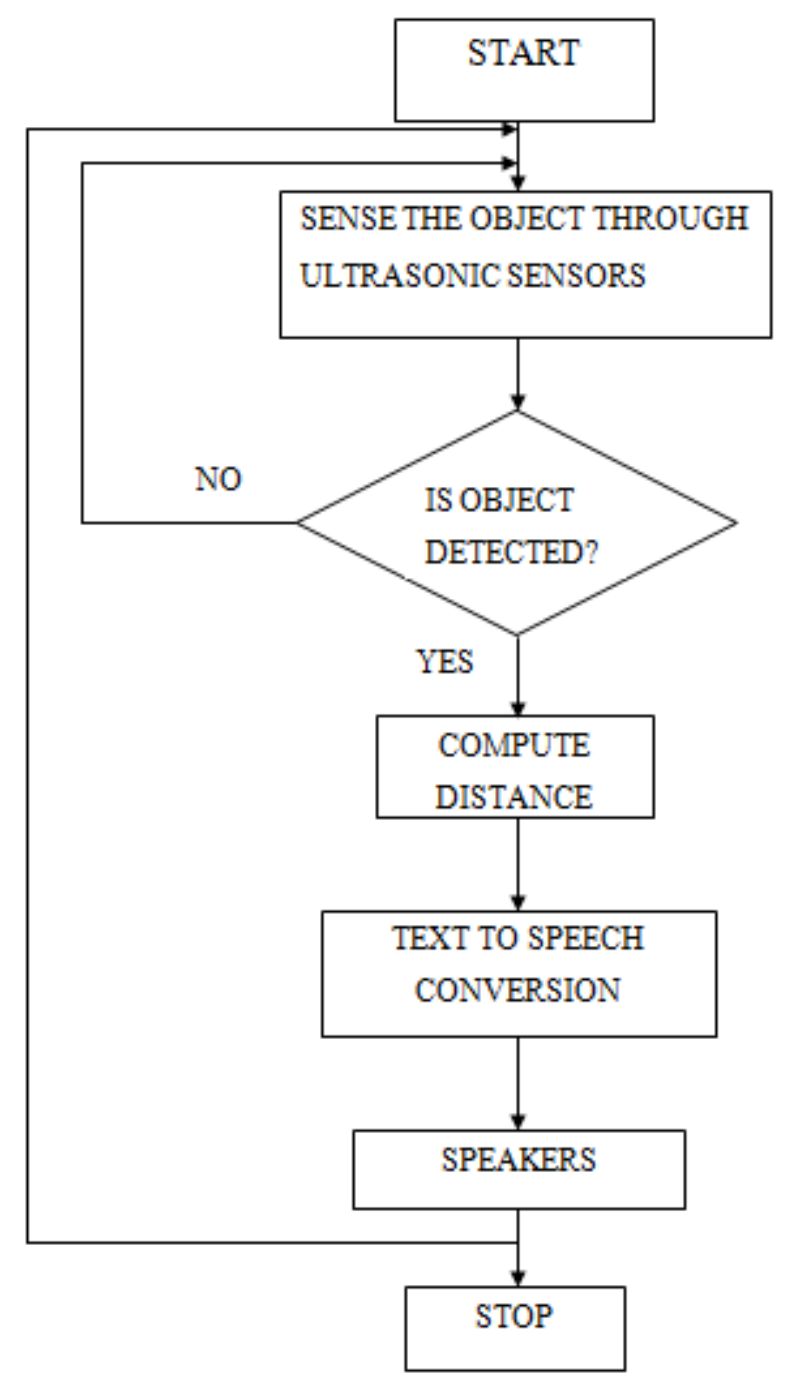

Fig. 4 Flowchart of smart electronic stick

Figure 4 shows the process, in which the first step is ultrasonic sensor senses the object, if the object is sensed then it computes the distance and if the length of the object to the person is less than or equal to $50 \mathrm{~cm}$ then it convert those signals to speech and send that speech message to the speakers, and finally the process ends.

The advantages of the proposed algorithm:

1. Low outline time.

2. Low creation cost.

3. This framework is material for both the indoor and open-air condition.

4. It is a dynamic framework.

5. Low power consumption

\section{OBSTACLE DETECTION-TEST CASES}

The current developed unit was tested for various test cases and was given in the following as follows, 
Example 1: If the reflected signals are from the first sensor then microcontroller retrieves the respective audio file from SD card and sends output to the speakers as "obstacle please turn left".

Case 2: If the reflected signals are from the second sensor then microcontroller retrieves the respective audio file from SD card and sends output as "obstacle please turn right".

Case 3: If the reflected signals are from the third sensor then microcontroller retrieves the respective audio file from SD card and sends output to the speakers as "obstacle please move forward immediately".

Case 4: If the reflected signals are from the fourth sensor then microcontroller retrieves the respective audio file from SD card and sends output as "obstacle please come back".

Case 5: If two or more sensors sense the obstacle then microcontroller retrieves the audio file from SD card and sends output to the speakers as "obstacle".

Case 6: If left and right sensors sense the obstacle then microcontroller retrieves the audio file from SD card and sends output to the speakers as "obstacle in left and right".

Case 7: If right and front sensors sense the obstacle then microcontroller retrieves the audio file from SD card and sends output to the speakers as "obstacle in right and front".

Case 8: If front and back sensors sense the obstacle then microcontroller retrieves the audio file from SD card and sends output to the speakers as "obstacle in front and back".

Case 9: If back and left sensors sense the obstacle then microcontroller retrieves the audio file from SD card and sends output to the speakers as "obstacle in back and left".

Case 10: If left and front sensors sense the obstacle then microcontroller retrieves the audio file from SD card and sends output to the speakers as "obstacle in left and front".

Case 11: If back and right sensors sense the obstacle then microcontroller retrieves the audio file from SD card and sends output to the speakers as "obstacle in back and right".

Case 12: If left, right and front sensors sense the obstacle then microcontroller retrieves the audio file from SD card and sends output to the speakers as "obstacle in left, right and front".

Case 13: If right, back and front sensors sense the obstacle then microcontroller retrieves the audio file from SD card and sends output to the speakers as "obstacle in right, back and front".

Case 14: If front, back and left sensors sense the obstacle then microcontroller retrieves the audio file from SD card and sends output to the speakers as "obstacle in front, back and left".

Case 15: If back, left and right sensors sense the obstacle then microcontroller retrieves the audio file from SD card and sends output to the speakers as "obstacle in the back, left and right". 
Obstacle Awareness: The extent of the obstacles on the test course recognised by the visually impaired user. A higher number demonstrates expanded observation of nature while exploring.

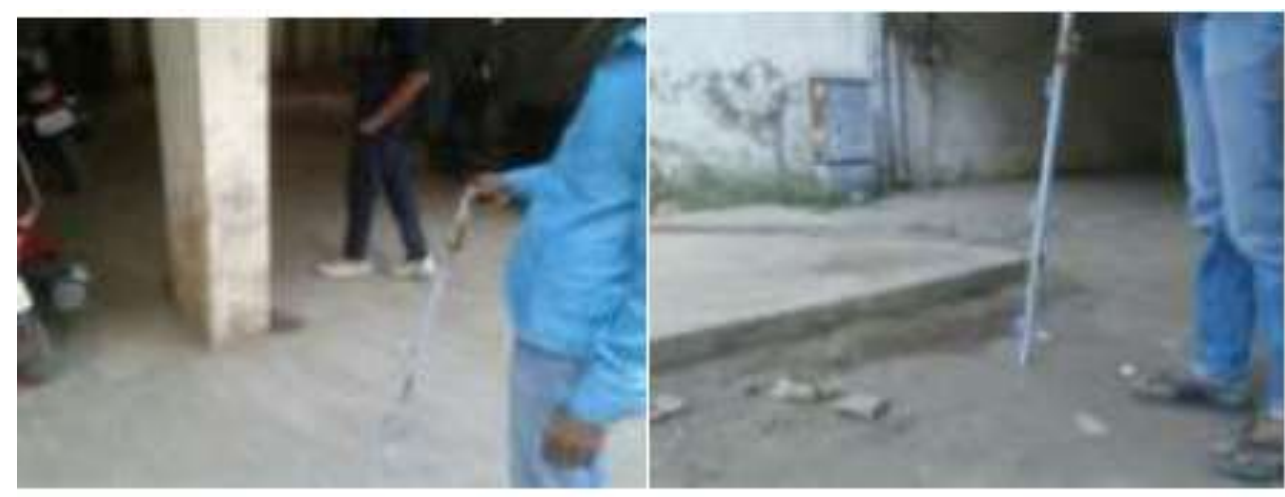

Fig. 5 Obstacle Detection such as the poll, steps

Collision rate: The extent of obstacles impacts per number of barriers experienced. A lower collision rate shows expanded security for the user.
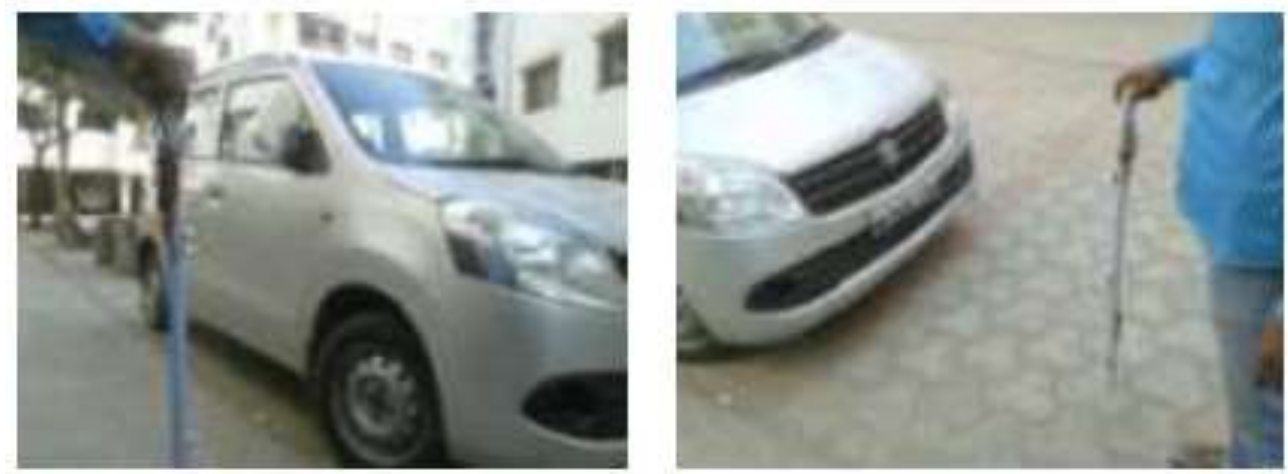

Fig. 6 Obstacle Detection such as car

\section{CONCLUSION}

A basic, simple, easy configurable electronic direction framework is proposed to give helpful colleague and support to blind and visually impaired people. The structure is composed, actualised, tried and checked. The ongoing consequences of the framework are empowering and it uncovered a precision of $93 \%$ in recognising separations. The outcomes demonstrate that the structure is proficient and one of a kind in its ability in determining the source and separation of the items that may experience the visually impaired. It can filter territories left, appropriate, back and before the visually impaired individual paying little respect to its stature or profundity. Accordingly, it was supported by the individuals who took an interest in the test. The ultrasonic sensor had been entirely used to propel the portability of the visually impaired and visual hindered individuals in sheltered and freeway. This framework does not require a great gadget to behold for a long separation and it does not need any exceptional preparing.

\section{REFERENCES}

[1] Amjed S. Al-Fahoum, Heba B. Al-Hmoud, and Australia A. Al- Fraihat, "A Smart Infrared Microcontroller-Based Blind Guidance System", Hindawi Transactions on Active and Passive Electronic Components, Vol.3, No.2, pp.1-7, June 2013. 
[2] Harshad Girish Lele, Viten Vilas Lonkar, Varun Vasant Marathe and Mrunmayi Mohan Modak, "Electronic Path Guidance for Visually Impaired People", The International Journal of Engineering and Science (IJES), Vol.2, No.4, pp.9-12, April 2013.

[3] C. Watters, M. Owen, and S. Munroe, "A study of blind demographics and services in Canada," Tech. Rep., 2004.

[4] A. Meade, "Dexter-A finger-spelling hand for the blind," in Proceedings. 1987 IEEE International Conference on Robotics and Automation, vol. 4. Institute of Electrical and Electronics Engineers, 1987, pp. 1192-1195.

[5] S. Eberhardt, L. Bernstein, D. Coulter, and L. Hunckler, "OMAR haptic braille for blind individuals", in Proceedings of IEEE Virtual Reality Annual International Symposium. IEEE, 1993, pp. 195-201.

[6] K. Durre, "BrailleButler: a new approach to non-visual computer applications," in [1990] Proceedings. Third Annual IEEE Symposium on Computer-Based Medical Systems. IEEE Comput. Soc. Press, 1990, pp. 97-104.

[7] N. Sriskanthan and K. Subramanian, "Braille display terminal for personal computers," IEEE Transactions on Consumer Electronics, vol. 36, no. 2, pp. 121-128, May 1990.

[8] M. Bazzani and E. Mumolo, "PC-based telephone communications system for deaf-blind people," in IEEE Global Telecommunications Conference and Exhibition. Communications for the Information Age. IEEE, 1988, pp. 43-47. 
International Journal of Security and Its Applications Vol. 13, No. 1 (2019) 